\title{
Differential effects of saturated versus unsaturated dietary fatty acids on weight gain and myocellular lipid profiles in mice
}

\author{
S Timmers ${ }^{1,2}$, J de Vogel-van den Bosch ${ }^{1,2}, \mathrm{~N}_{\text {de Wit }}{ }^{1,3}, \mathrm{G} \mathrm{Schaart}^{4}$, D van Beurden ${ }^{2}, \mathrm{M} \mathrm{Hesselink}^{4}$, \\ $\mathrm{R}$ van der Meer ${ }^{1,3}$ and P Schrauwen ${ }^{1,2}$
}

${ }^{1}$ Top Institute Food and Nutrition (TIFN), Wageningen, The Netherlands; ${ }^{2}$ Department of Human Biology, School for
Nutrition, Toxicology and Metabolism (NUTRIM), Maastricht University Medical Centre, Maastricht, The Netherlands;
${ }^{3}$ Nutrition, Metabolism and Genomics Group, Division of Human Nutrition, Wageningen University, Wageningen, The
Netherlands and ${ }^{4}$ Department of Human Movement Sciences, School for Nutrition, Toxicology and Metabolism (NUTRIM),
Maastricht University Medical Centre, Maastricht, The Netherlands

Objective: In conditions of continuous high-fat (HF) intake, the degree of saturation of the fatty acids (FAs) in the diet might have a crucial role in the onset of obesity and its metabolic complications. In particular, the FA composition of the diet might influence the storage form of lipids inside skeletal muscle. The aim of the present study was to examine whether the FA composition of $\mathrm{HF}$ diets differentially affects weight gain and accumulation of myocellular triacylglycerol (TAG) and diacylglycerol (DAG). Furthermore, we examined whether the FA composition of the diet was reflected in the composition of the myocellular lipid intermediates.

Design: C57Bl6 mice were fed HF diets (45\% energy) mainly containing palm oil (PO), cocoa butter (CB), olive oil (OO) or safflower oil (SO; $n=6$ per group) for 8 weeks. A low-fat diet (10\% energy, PO) was used as control. Body weight was monitored weekly. At the end of the dietary intervention, myocellular TAG and DAG content and profiles were measured.

Results: We here show that HF_CB prevented weight gain after 8 weeks of HF feeding. Furthermore, the HF diet rich in SO prevented the accumulation of both myocellular TAG and DAG. Interestingly, the FA composition of DAG and TAG in skeletal muscle was a reflection of the dietary FA composition.

Conclusion: Already after a relatively short period, the dietary FA intake relates to the FA composition of the lipid metabolites in the muscle. A diet rich in polyunsaturated FAs seems to prevent myocellular lipid accumulation.

Nutrition and Diabetes (2011) 1, e11; doi:10.1038/nutd.2011.7; published online 11 July 2011

Keywords: high-fat feeding; fatty acid composition; intramyocellular lipids; diacylglycerol; glucose tolerance

\section{Introduction}

Obesity is a serious health problem, predisposing individuals to a range of metabolic complications. Skeletal muscle and adipose tissue are important organs in regulating fatty acid (FA) metabolism, and disturbances in their function may lead to insulin resistance and dyslipidemia. ${ }^{1}$ When the capacity of adipose tissue to store lipid becomes insufficient, which is most often associated with chronic excessive energy intake (in particular by means of dietary fat), this may lead to

Correspondence: Professor P Schrauwen, Department of Human Biology, Maastricht University Medical Centre, PO Box 616, Maastricht 6200 MD, The Netherlands.

E-mail: p.schrauwen@maastrichtuniversity.nl

Received 17 November 2010; revised 4 April 2011; accepted 23 May 2011 an increased supply of triacylglycerols (TAGs) and free FAs to non-adipose tissues, such as skeletal muscle and liver. ${ }^{1}$ Consequently, if the uptake of FAs exceeds the capacity or the need to oxidize fat in skeletal muscle or liver, the FAs are stored. Indeed, TAG content in human skeletal muscle has been reported to increase by $36-90 \%$ after high-fat (HF) feeding periods ranging from $24 \mathrm{~h}$ to 7 weeks, ${ }^{2-5}$ and numerous studies have shown a negative association between fat accumulation in these tissues and insulin sensitivity. ${ }^{6-9}$

Next to total fat content, it has nowadays become apparent that dietary fat quality (that is, dietary FA composition) may also be important, as each individual FA has distinct chemical properties linked to its unique structure. Thus, it has been shown that the type of dietary fat can influence the onset of obesity by differentially 
affecting weight gain and body fatness. Saturated FAs (SFAs) have been shown to result in higher rates of weight gain, ${ }^{10,11}$ whereas polyunsaturated FAs (PUFAs) did not induce obesity in mice and rats. ${ }^{12,13}$ In humans, Doucet et al. ${ }^{14}$ showed that waist circumference, as a measure of central adiposity, is increased in individuals with a higher total, saturated and monounsaturated fat content in their diets compared with PUFAs.

Furthermore, the degree of saturation of the diet has been suggested to affect the rate of storage of FA intermediates. Both in rats and humans, it was shown that unsaturated FAs are oxidized more rapidly than SFAs, ${ }^{15,16}$ and SFAs, which are less readily oxidized, accumulate as diacylglycerols (DAGs) and ceramide in vitro, ${ }^{17}$ whereas monounsaturated FAs (MUFAs) and PUFAs are efficiently stored as TAGs. ${ }^{17-19}$

Despite these suggestions that dietary fat quality can affect body weight gain and muscle fat storage, no studies are available that consistently tested the effect of well-controlled experimental $\mathrm{HF}$ diets varying in FA composition on myocellular lipid content. Moreover, whereas it has been shown for adipose tissue that TAG composition reflects habitual dietary FA intake over a considerably long time, ${ }^{20,21}$ such information is lacking for skeletal muscle.

Therefore, we investigated whether the FA composition of HF diets, with different degree of saturation, differentially affects body weight gain and storage of dietary FAs into either myocellular TAG or DAG. Furthermore, we were interested whether, after a short period of change in dietary fat intake, the FA profiles of myocellular TAG and DAG represent the dietary FA composition.

\section{Materials and methods}

\section{Animals and diets}

Thirty, male C57Bl/6J mice, aged 4 weeks, were obtained from Harlan Laboratories (Horst, The Netherlands) and were acclimatized, two per cage, to the environmentally controlled laboratory environment (temperature $22 \pm 1{ }^{\circ} \mathrm{C}$ and relative humidity $55 \pm 2 \%$ ) with a 12:12 light-dark cycle. Three weeks before the onset of the experimental intervention, mice were allowed ad libitum access to a low-fat diet (LF_PO, palm oil; $70 \%, 10 \%$ and $20 \%$ of calories as carbohydrate, fat and protein, respectively) that contained both SAT FAs and MUFAs derived from PO (Research Diet Services, Wijk bij Duurstede, The Netherlands).

After these 3 weeks, mice were randomly assigned to one of the six experimental diet groups for a period of 8 weeks. The first group was maintained on the LF_PO $(n=6)$. The other mice switched to a HF diet (35\% carbohydrate; $45 \%$ fat; and $20 \%$ protein) that consisted mainly of SFAs, derived from cocoa butter (HF_CB, $n=6$ ), a mixture of both SFAs and MUFAs, derived from PO (HF_PO, $n=6)$, two mixtures primarily of MUFAs and PUFAs, derived from olive oil (HF_OO, $n=6$ ) and safflower oil (HF_SO, $n=6$ ) (Research
Table 1 Macronutrient composition of the diets

\begin{tabular}{|c|c|c|c|c|c|}
\hline & $\begin{array}{c}L F_{-} P O \\
D 12450 B\end{array}$ & $\begin{array}{c}H F \_P O \\
D 12451\end{array}$ & $\begin{array}{c}H F_{-} C B \\
D 12451\end{array}$ & $\begin{array}{c}\text { HF_OO } \\
\text { D12451 }\end{array}$ & $\begin{array}{c}\text { HF_SO } \\
\text { D12451 }\end{array}$ \\
\hline \multicolumn{6}{|l|}{ Based on formula } \\
\hline Protein (kcal \%) & 20 & 20 & 20 & 20 & 20 \\
\hline Carbohydrate (kcal \%) & 70 & 35 & 35 & 35 & 35 \\
\hline Fat (kcal \%) & 10 & 45 & 45 & 45 & 45 \\
\hline Total & 100 & 100 & 100 & 100 & 100 \\
\hline \multicolumn{6}{|l|}{ Percentage of fatty acids } \\
\hline $\mathrm{C} 16: 0$ & 43 & 43 & 25 & 12 & 6 \\
\hline C18:0 & 4 & 4 & 34 & 2 & 3 \\
\hline C18:1 & 41 & 41 & 33 & 73 & 12 \\
\hline C18:2 & 10 & 10 & 3 & 10 & 78 \\
\hline
\end{tabular}

Abbreviations: HF_CB, high-fat cocoa butter; HF_OO, high-fat olive oil; HF_PO, high-fat palm oil; HF_SO, high-fat safflower oil; LF_PO, low-fat palm oil.

Diet Services). The exact composition of the diets is given in Table 1. Animals were provided with food and water ad libitum throughout the experimental period. Body weight of the mice was monitored weekly during the dietary intervention period. To determine food intake, non-absorbable chromic oxide was supplemented to the diets for 1 week (week 5 of the diet intervention). At the end of this week, feces were quantitatively collected during $48 \mathrm{~h}$ and fecal chromic oxide levels were determined as previously described. ${ }^{22}$ These fecal chromic oxide levels were then used to calculate the energy intake per mouse per day.

All experiments were approved by the Institutional Animal Care and Use committee of the Wageningen University and complied with the principles of laboratory animal care.

\section{Oral glucose tolerance test}

To investigate the effect of dietary FA composition on wholebody glucose tolerance, mice underwent an oral glucose tolerance test after 7 weeks of the dietary intervention. After a 6-hour fast, glucose (20\% wt/vol solution) was administered by oral gavage at a dose of $2.5 \mathrm{~g} / \mathrm{kg}$ body weight, and glucose was measured in blood obtained from the tail vein, before glucose administration $(t=0)$ and at 15, 30, 45, 60 and 90 min thereafter using Accu-Chek blood glucose meters (Roche Diagnostics, Almere, The Netherlands). Incremental area under the curve of glucose concentration versus time curve was calculated using the trapezoidal method.

\section{Tissue sampling}

After 8 weeks of dietary intervention, mice were anaesthetized in the postprandial phase at 1400 hours with a mixture of isoflurane, nitrogen oxide and oxygen, followed by cervical dislocation. Gastrocnemius muscle was rapidly dissected out, immediately frozen in liquid nitrogen-cooled isopentane and stored at $-80^{\circ} \mathrm{C}$ until further analyses. Epididymal fat depots were isolated and weighed. 
Measurement of skeletal muscle TAG and DAG content Total lipids were extracted from frozen muscle using the method of Folch et al. ${ }^{23}$ The extracts were filtered and lipids recovered in the chloroform phase. Total TAGs and DAGs were isolated using thin-layer chromatography on Silica Gel 60 A plates (Merck kGaA, Darmstadt, Germany) developed in petroleumbenzin-diethyl ether-acetic acid (120:25:1.5 by volume) and visualized by rhodamine 6G. The TAG and DAG bands were scraped from the plate and methylated using a mixture of toluene-methanol (BF3/methanol 14\%; $20-55-25 \%$ by volume), as described by Morrison and Smith. ${ }^{24}$ The methylated FAs were extracted with hexane and analyzed by capillary gas liquid chromatography using a $50 \mathrm{~m} \times 0.25 \mathrm{~mm}$ CP-sil 88 silica column (Varian, Agilent, Santa Clara, CA, USA), with helium as carrier gas at a flow of $130 \mathrm{kPa}$. The column oven was maintained at $165^{\circ} \mathrm{C}$ for $10 \mathrm{~min}$ and increased at a rate of $5^{\circ} \mathrm{C} \mathrm{min}^{-1}$ to $190^{\circ} \mathrm{C}$. This temperature was maintained for $15 \mathrm{~min}$. Then temperature was increased to $230^{\circ} \mathrm{C}$ for $22 \mathrm{~min}$, with a flow rate of $2{ }^{\circ} \mathrm{Cmin}^{-1}$. FA methyl esters were identified by comparing retention times to those of known standards. Inclusion of an internal standard, 17:0 (heptadecanoic acid, internal standard for TAG), 19:0 (dinonadecanoic acid, internal standard for DAG) and an average molecular mass for each FA methyl ester permits quantification of the amount of TAG and DAG in the sample, expressed as micromolar per gram of wet tissue weight. Contribution of the individual FAs as percentage of total DAG and TAG content was calculated.

\section{Enzyme activity measurements}

Muscle $\beta$-hydroxyacyl-CoA dehydrogenase and citrate synthase, and enzyme activities were determined spectrophotometrically. In short, $30(20 \mu \mathrm{m})$ sections of muscle were added to $100 \mu \mathrm{l}$ sucrose-EDTA-Tris buffer $\left(250 \mathrm{mmol}^{-1}\right.$ sucrose, $2 \mathrm{mmoll}^{-1}$ EDTA and $10 \mathrm{mmoll}^{-1}$ Tris ( $\left.\mathrm{pH} 7.4\right)$ ) and sonicated (Branson 2210; Branson Ultrasonics, Danbury, CT, USA) for $25 \mathrm{~s}$. Samples were centrifuged (10 min, $10000 \mathrm{~g}$, $4{ }^{\circ} \mathrm{C}$ ), and the supernatants were analyzed spectrophotometrically (Multiskan Spectrum; Thermo labsystems, Breda, The Netherlands) to determine $\beta$-hydroxyacyl-CoA dehydrogenase $^{25}$ and citrate synthase enzyme activities. ${ }^{26}$ Activity of proteins is expressed as $\mathrm{Ug}^{-1}$ protein.

\section{Measurement of liver fat content}

Animals were killed and livers were rapidly excised, rinsed of blood and homogenized by polytron (Brinkman Instruments, Westbury, NY, USA) in homogenization buffer (containing $10 \mathrm{~mm}$ Tris, $2 \mathrm{~mm}$ EDTA and $0.25 \mathrm{M}$ sucrose, $\mathrm{pH} 7.5,4{ }^{\circ} \mathrm{C}$ ). Triglyceride concentration was determined by the commercially available enzymatic kit (Instruchemie, Delfzijl, The Netherlands). Briefly, homogenates were centrifuges at $12000 \mathrm{~g}$ for $10 \mathrm{~min}$, and TAG amounts in the supernatants were determined by enzyme-linked immunosorbent assay. The TAG content was corrected for tissue weight.

\section{Western blotting}

Muscle samples were homogenized as described previously ${ }^{27}$ and processed for standard SDS-polyacrylamide gel electrophoresis and western blotting. Protein concentration was assessed, and equal amounts of protein were loaded per lane. Actin was used as a loading control. Membranes were incubated with antibodies against oxidative phosphorylation proteins (OXPHOS) (MS601; MitoSciences, Eugene, OR, USA), peroxisome proliferator-activated receptor-gamma coactivator 1 alpha (PGC1 $\alpha$ ) (516557; Calbiochem; VWR International BV, Amsterdam, The Netherlands) and adipose tissue triglyceride lipase (ATGL) (2138; Cell Signaling Technology; Bioké; Leiden, The Netherlands). Blots incubated with OXPHOS and PGC1 $\alpha$ were probed with IRDye800-conjugated or IRDye700-conjugated secondary antibodies (Rockland, Gilbertsville, PA, USA, and LICOR Biosciences, Westburg, Leusden, The Netherlands), and bands at a molecular weight corresponding to the control samples were quantified using the Odyssey infrared imaging system (LICOR Biosciences, Wateringen, The Netherlands). Blots detecting ATGL were probed with the appropriate horse radish-conjugated antibodies, and after incubation, specific protein bands were visualized by chemiluminescence and analyzed by Chemidoc XRS system (Bio-Rad, Veenendaal, The Netherlands).

\section{Statistical analysis}

Results are presented as the mean \pm s.e. Differences between groups were analyzed by one-way analysis of variance, with least significant differences post hoc correction. The accepted level of statistical significance was $P<0.05$ for all analyses. All calculations were done using the Statistical Package for Social Sciences (SPSS 16.0 software, SPSS, Chicago, IL, USA).

\section{Results}

Body weight, food intake, feces production and epididymal fat pads

Body weights gradually increased over the 8 weeks of intervention in all dietary groups (Figure 1). However, mice receiving HF_PO gained the most weight, whereas LF_PO and HF_CB mice displayed the lowest weight gain over the entire feeding protocol. The delta body weight was significantly different between groups $(+43 \%$ in HF_PO versus LF_PO, $P=0.001 ;+41 \%$ in HF_PO versus HF_CB, $P=0.001$; $+20 \%$ in HF_PO versus HF_OO, $P>0.05$; and $+26 \%$ in HF_PO versus HF_SO, $P=0.030)$. Food intake between the different dietary groups was not different (LF_PO, 12.4 \pm 1.4 ; HF_PO, $12.1 \pm 1.4 ; \mathrm{HF} \_\mathrm{CB}, 10.0 \pm 0.4$; HF_OO, $12.9 \pm 1.1$; and HF_SO, $15.1 \pm 0.7 \mathrm{kcal}$ per day, $P>0.05)$. Feces production was significantly higher for HF_CB compared with the other dietary groups (LF_PO, $0.40 \pm 0.02$; HF_PO, $0.41 \pm 0.03$; HF_CB, $0.53 \pm 0.01$; HF_OO, $0.39 \pm 0.01$; and HF_SO, $0.42 \pm 0.02 \mathrm{~g}$ per day, $P<0.05)$. Epididymal fat pad weights were not different between the dietary groups (LF_PO, 
$1.31 \pm 0.15 ; \mathrm{HF} \_P O, 2.14 \pm 0.13 ; \mathrm{HF} \_\mathrm{CB}, 1.74 \pm 0.15 ; \mathrm{HF} \_\mathrm{OO}$, $1.72 \pm 0.28$; and HF_SO, $1.71 \pm 0.25 \mathrm{~g}, P>0.05)$.

\section{Muscle lipid content}

After 8 weeks of dietary intervention, skeletal muscle TAG and DAG content were assessed.

Skeletal muscle TAG content increased to the same extent in all HF diets compared with LF_PO $(P<0.05$, Figure $2 \mathrm{a})$, except for HF_SO, which did not lead to accumulation of myocellular TAG.

HF_PO, HF_CB and HF_OO also significantly increased myocellular DAG levels compared with LF_PO $(P<0.05)$, whereas HF_SO did not significantly increase DAG content $(P=0.39$, Figure $2 b)$.

Dietary FA composition exerted a profound effect on the FA profile in skeletal muscle DAG and TAG (Figures 3a and b). Both LF_PO and HF_PO, which contain an almost equal amount of SFAs (49\% of total FAs, Table 1) and MUFAs (41\% of total FAs), increased the proportion of the SFA C16:0 and the MUFA C18:1 in both DAG and TAG. Also, HF_OO and HF_SO, two diets that are characterized by a high amount of MUFAs (73\% of total FAs) and PUFAs (78\% of total FAs), respectively, elevated the proportion of the unsaturated

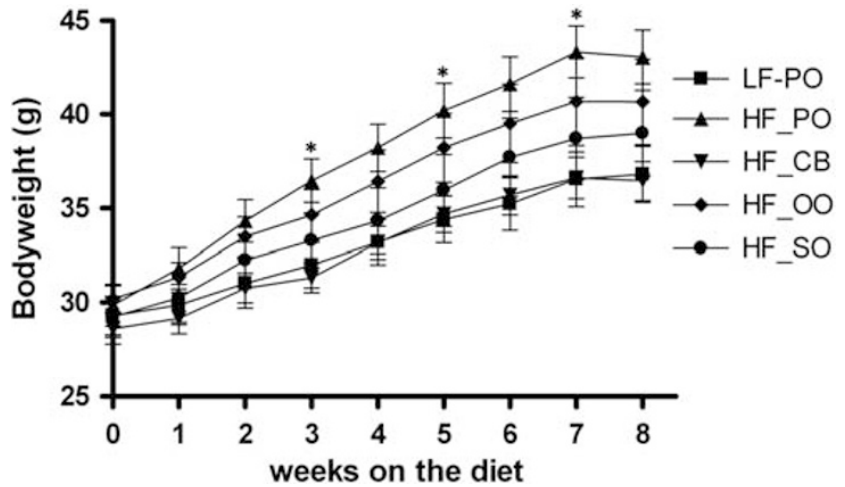

Figure 1 Effect of dietary FA composition on body weight gain. Body weight gradually increased over the 8 weeks of dietary intervention. Data are expressed as mean \pm s.e.m. $(n=6)$. ${ }^{*} P<0.05$ HF_PO versus LF_PO and HF_CB.
FAs in both skeletal muscle DAG and TAG content. For HF_OO, the increased proportion of MUFAs resulted in an increase in C18:1, whereas for HF_SO the elevated PUFAs in DAG and TAG content were due to an increase in C18:2. HF_CB, a diet consisting of $63 \%$ of SFAs and of $37 \%$ of unsaturated FAs, elevated both the proportion of C18:0 and C18:1 in skeletal muscle DAG and TAG content.

Glucose tolerance

Fasting blood glucose values were not affected by the FA composition of the different diets (LF_PO, 10.18 \pm 0.40 ;
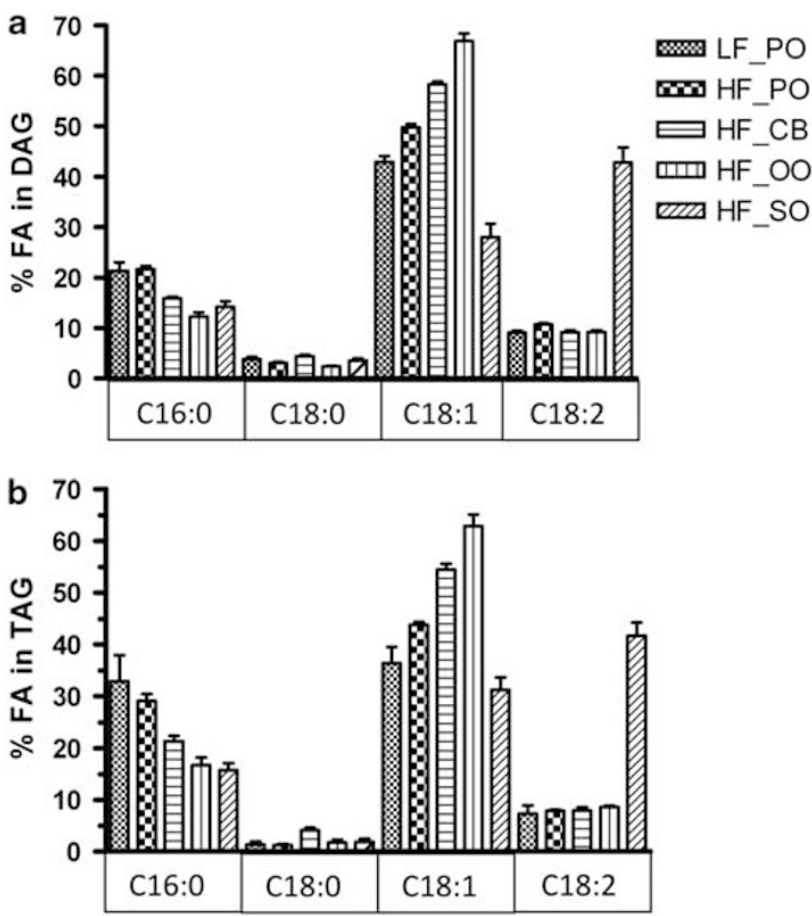

Figure 3 Effect of dietary FA composition on skeletal muscle DAG (a) and TAG (b) FAs. Representation of the FAs that account for the highest percentage in the diet and account, on average, for $80 \%$ of total DAG and TAG content. Data are expressed as mean \pm s.e.m. $(n=6)$.
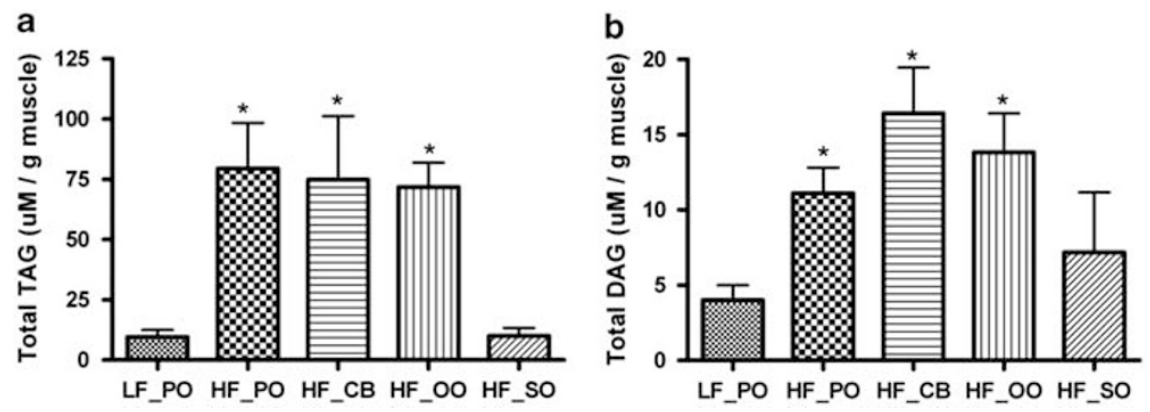

Figure 2 Effect of dietary FA composition on TAG (a) and DAG (b) content in gastrocnemius muscle. Data are expressed as mean \pm s.e.m. $(n=6)$. $P<0.05$ LF_PO versus HF_PO, HF_CB and HF_OO. 
HF_PO, $12.14 \pm 0.84 ; \quad$ HF_CB, $11.0 \pm 0.23$; HF_OO, $11.5 \pm 1.59$; and HF_SO, $10.88 \pm 0.65 \mathrm{mM}, P>0.05)$.

The degree of saturation of the different HF diets had no major influence on whole-body glucose tolerance, that is, all the HF diets reduced the clearance of glucose from the blood compared with the LF_PO (Figures $4 \mathrm{a}$ and b), although this did not reach statistical significance for HF_OO $(+45.6 \%$ in HF_PO versus LF_PO, $P<0.005$; $+38.5 \%$ in HF_CB versus LF_PO, $P<0.022$; $+28.9 \%$ in HF_OO versus LF_PO, $P=0.16$; and $+45.4 \%$ in HF_SO versus LF_PO, $P<0.003)$. No significant differences between different HF groups were evident. We found a positive correlation between the incremental area under the blood glucose curve and
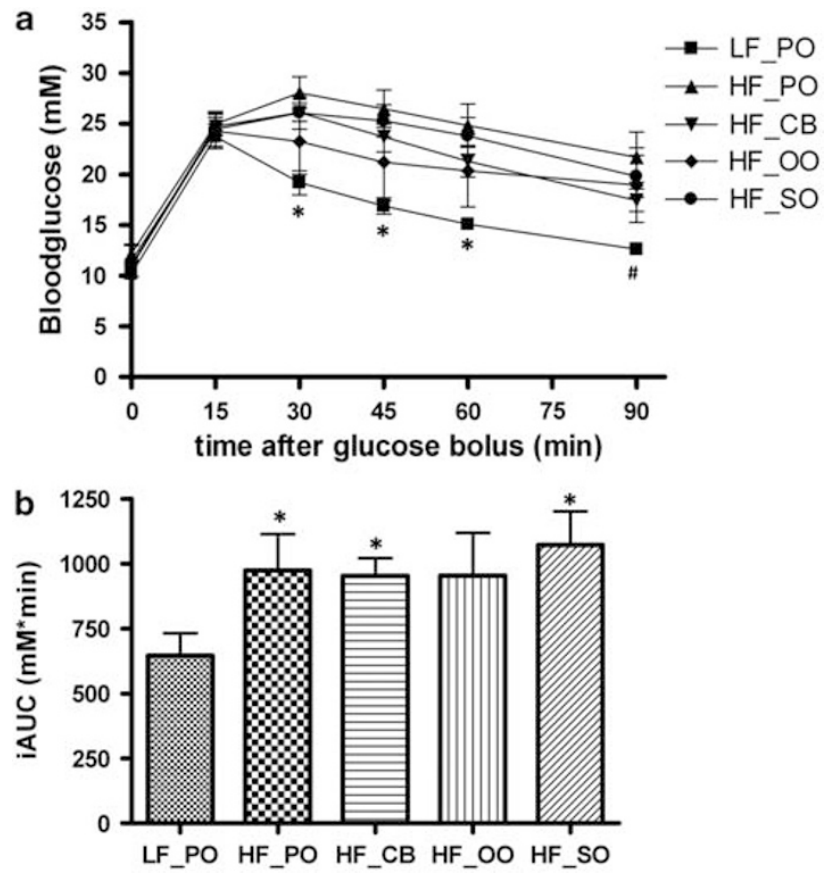

Figure 4 Effect of dietary FA composition on whole-body glucose tolerance. Glucose clearance after oral glucose bolus $\left(2.5 \mathrm{~g} \mathrm{~kg}^{-1} ; \mathrm{a}\right)$ and area under the curve (AUC) of the glucose tolerance test (b). Data are expressed as mean \pm s.e.m. $(n=6) .{ }^{*} P<0.05$ LF_PO versus HF_PO, HF_CB and HF_SO. ${ }^{\#} P<0.05$ LF_PO versus HF_PO and HF_SO. myocellular DAG and TAG content (Pearson correlation coefficient $=0.35, P=0.10$, Figure $5 \mathrm{a}$; Pearson correlation coefficient $=0.43, P=0.039$, Figure $5 b$, respectively).

Skeletal muscle insulin signaling

Skeletal muscle IRS ser ${ }^{307}$ phosphorylation, a marker of lipidinduced insulin resistance, was similar between all dietary groups (LF_PO, $0.81 \pm 0.04$; HF_PO, $1.25 \pm 0.19$; HF_CB, $0.96 \pm 0.14 ;$ HF_OO, $0.82 \pm 0.09$; and HF_SO, $0.89 \pm 0.09 \mathrm{AU}$, $P>0.05)$.

GLUT4 protein content in sarcolemma was not affected by the composition of the HF diets (LF_PO, $0.94 \pm 0.14$; HF_PO, $1.05 \pm 0.09$; HF_CB, $0.98 \pm 0.14 ;$ HF_OO, $1.08 \pm 0.20$; and HF_SO, $1.09 \pm 0.11 \mathrm{AU}, P>0.05)$.

\section{Liver lipid content}

As liver is also an important organ involved in glucose tolerance, liver fat content was also measured. Liver TAG content was increased in mice receiving HF_PO and HF_OO compared with mice receiving LF_PO, HF_CB and HF_SO after 8 weeks of HF feeding $(P<0.05$, Figure 6$)$.

Muscle oxidative capacity

We also examined whether the composition of the HF diets lead to alterations in muscle oxidative capacity. Citrate synthase activity in whole-muscle homogenate was similar between the LF and the different HF groups (LF_PO, $21.88 \pm 1.59$; HF_PO, $21.33 \pm 1.12$; HF_CB, $19.10 \pm 0.89$; HF_OO, 22.93 \pm 2.23 ; and HF_SO, $21.49 \pm 0.94 \mathrm{U} \mathrm{g}^{-1}$ protein, $P>0.05)$. No differences in $\beta$-hydroxyacyl-CoA dehydrogenase activity were detected between the different dietary groups, indicating similar $\beta$-oxidative capacity (LF_PO, $34.70 \pm 4.79$; HF_PO, $37.95 \pm 3.10$; HF_CB, 37.17 \pm 4.50 ; HF_OO, $36.98 \pm 6.75$; and HF_SO, $35.64 \pm 6.47 \mathrm{Ug}^{-1}$ protein, $P>0.05$ ).

Eight weeks of HF feeding did not decrease PGC1 $\alpha$ protein levels, a strong marker of mitochondrial biogenesis (Figure 7a). Moreover, mitochondrial density was not statistically different between diets as determined by western blotting of the structural complexes of the respiratory chain
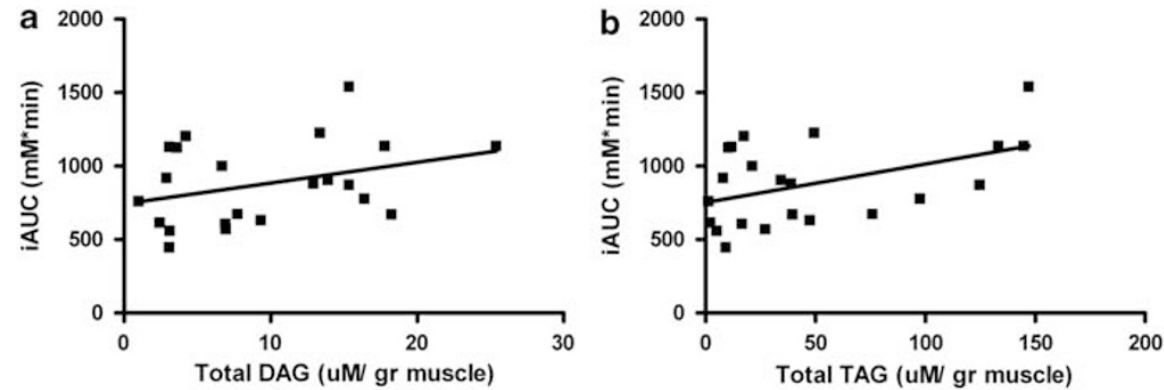

Figure 5 Relation between glucose intolerance and total muscular DAG (a) and TAG (b) content. Correlations $(r=0.35$ and $P=0.10, r=0.43$ and $P=0.039$ for DAG and TAG, respectively) are linear. 
in whole-muscle homogenate (Figures 7b-e). Furthermore, protein levels of ATGL, the main lipase in the muscle responsible for the breakdown of TAG to DAG was

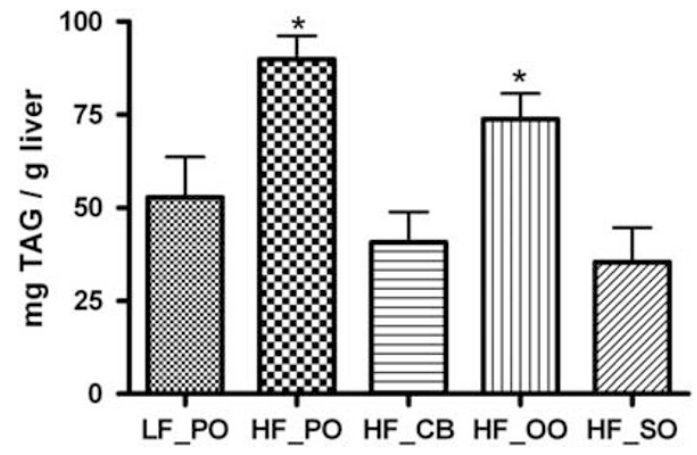

Figure 6 Effect of dietary FA composition on liver TAG content. Data are expressed as mean \pm s.e.m. $(n=6)$. ${ }^{*} P<0.05$ LF_PO, HF_CB and HF_SO versus HF_PO and HF_OO. unaffected by the composition of the different diets (LF_PO, $0.88 \pm 0.17$; HF_PO, $0.94 \pm 0.10$; HF_CB, $1.02 \pm 0.08$; HF_OO, $1.12 \pm 0.12$; and HF_SO, $1.08 \pm 0.12 \mathrm{AU}, P>0.05)$.

\section{Discussion}

From literature, it is known that in conditions of a continuous HF intake (that is, obesity), the degree of saturation of the FAs in the diet might have a crucial role in the development of obesity-related metabolic complications, as it might influence the storage form of FAs inside the muscle. Therefore, the present study examined whether diets varying in FA composition could differentially affect the rate of body weight gain and the accumulation of fat in skeletal muscle. Here, we show that after 8 weeks of HF feeding, only HF_CB prevented body weight gain. Furthermore, we show that a high dietary fat intake in the form of SO prevented the accumulation of fat in skeletal muscle. Interestingly, the FA
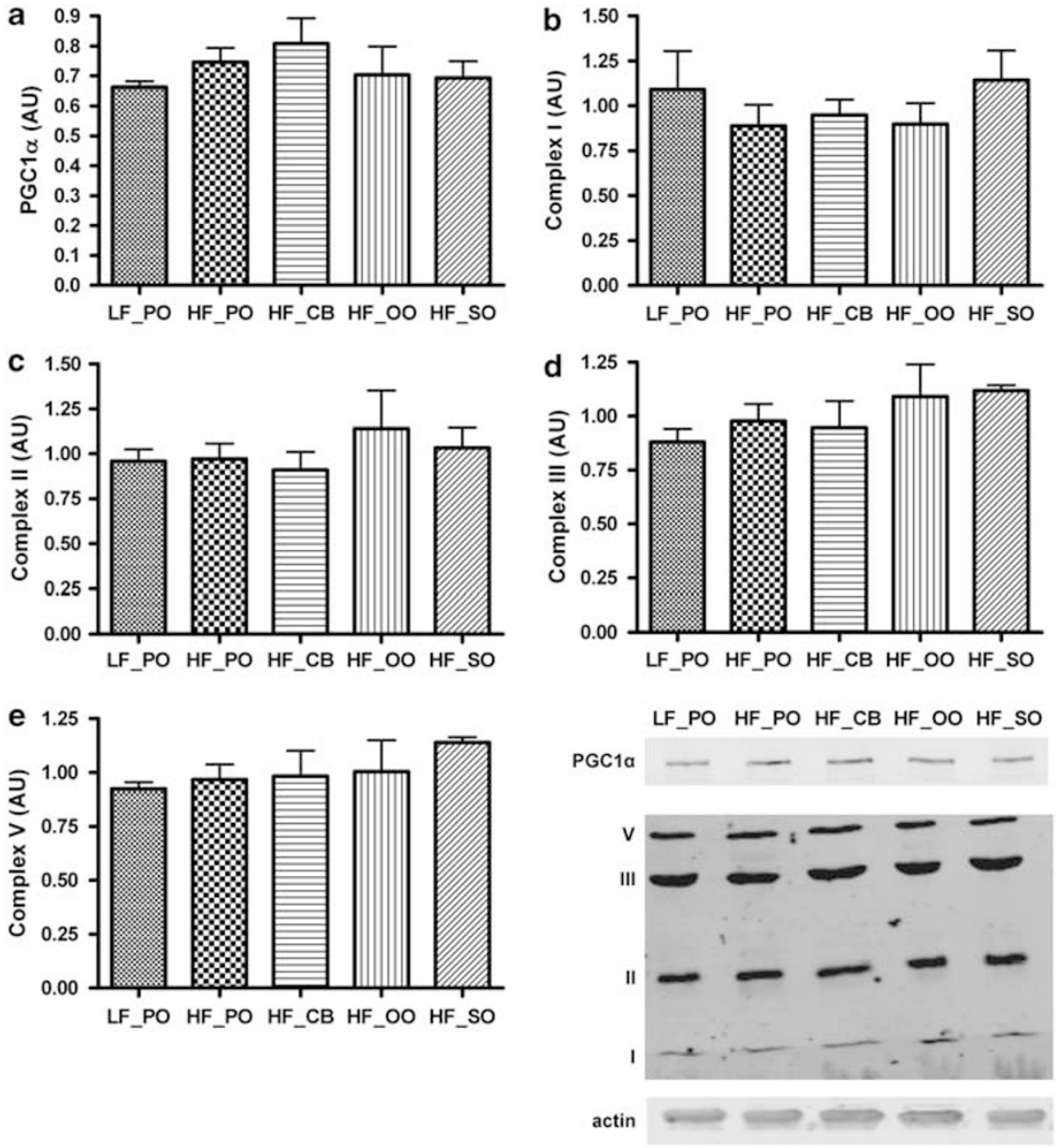

Figure 7 Effect of dietary FA composition on the expression of PGC1 $\alpha$ (a) and the individual complexes I, II, III and V of the respiratory chain (b-e) in gastrocnemius muscle. Data are expressed as mean \pm s.e.m. $(n=6)$. 
composition of DAG and TAG in skeletal muscle was a reflection of the dietary FA composition.

Eight weeks of HF feeding gradually increased body weight in all dietary groups. However, the change in body weight over the 8 weeks of dietary intervention varies between the different HF diets. This indicates that besides the high amount of fat, the type of FAs in the diet also influences the rate of weight gain in the development of obesity, as food intake was similar for all the dietary groups. Whereas HF_PO, which is rich in palmitic acid (43\% C16:0) and oleic acid (41\% C18:1), induced the highest increase in body weight during the dietary intervention period, HF_CB only led to a small increase in body weight, which was not significantly different from the LF_PO diet. The lower body weight gain for the HF_CB group can be explained by the fact that it is a vegetable fat that is rich in SFAs; palmitic acid (C16:0); and stearic acid (C18:0), thereby influencing the digestibility of the lipid. Animal studies have shown that 5-20\% CB in the diet results in lower digestibility (60-70\%), resulting in a higher fecal lipid elimination. ${ }^{28,29}$ In accordance, mice receiving the HF_CB diet also had a higher feces production. However, because food intake was only recorded during 1 week of the intervention, and feces was not analyzed for fat content, we cannot exclude the possibility that cumulative small, nonsignificant differences in food intake or a difference in fat partitioning may underlie the reduced body weight gain in these animals. Moreover, measurements of $24 \mathrm{~h}$ energy expenditure would have been helpful in interpreting energy and fat balance, but were not performed in the current study.

At the end of the dietary intervention, the storage of lipids inside the muscle for diets containing a high amount of SFAs (HF_CB), MUFAs (HF_OO) or a combination of both (HF_PO) were significantly increased compared with the LF_PO diet. Interestingly, for HF_SO, myocellular TAG levels were comparable to the LF_PO diet, indicating that a diet rich in PUFAs does not result in skeletal muscle lipid accumulation. For myocellular DAG accumulation, a similar pattern was observed, whereas the HF diets compromising mostly SFAs (HF_CB), MUFAs (HF_OO), or a combination of both types of FAs (HF_PO) significantly elevated myocellular DAG content, HF_SO, containing primarily PUFAs, did not increase DAG levels compared with the LF-fed animals.

These results indicate that diets rich in PUFAs may have beneficial effects on myocellular fat accumulation and are in line with reports showing a preferential oxidation of PUFAs, whereas SFAs appear to be predominantly stored in muscle rather than oxidized for energy. ${ }^{15,30}$ The finding that the HF_CB diet, which has by far the highest content of SFA and leads to a reduced fat absorbance and body weight increase, still increased DAG and TAG markedly, also suggests that SFAs that are taken up are redirected toward storage as TAGs and DAGs in skeletal muscle.

As it is well known that fat accumulation in muscle, especially also in the form of DAG, may lead to muscle insulin resistance, our data may also explain why dietary
MUFAs, and especially the PUFA linoleic acid (C18:2), have been linked to improved insulin sensitivity. ${ }^{31-33}$ In literature, the beneficial effect of PUFAs on insulin sensitivity is generally accounted for by a direct effect on insulin action rather than lowering glucose values. ${ }^{31}$ Unfortunately, we could not determine insulin sensitivity in our study, but we did find that whole-body glucose tolerance was positively correlated with myocellular DAG and TAG levels. We also measured makers of insulin sensitivity, such as IRS ser ${ }^{307}$ phosphorylation - an indicator of lipid-induced insulin resistance ${ }^{34}$ - and GLUT4 protein content of the sarcolemma but no changes were induced by the different dietary treatments. As muscle lipid content is not the only determinant of glucose tolerance, we also determined liver TAG content. Again, HF_SO did not increase liver TAG after 8 weeks of HF feeding. Furthermore, HF_CB also did not increase liver TAG. Further studies are necessary to examine whether changes in myocellular and hepatic lipid accumulation, induced by different types of dietary fat, will have functional consequences, which, however, was out of the scope of the present study.

We here also show that the dietary FA composition of the individual HF diets is reflected in the FA pattern of both myocellular TAG and DAG. Thus, the FA most represented in the individual HF diets also accounted for the highest percentage within both myocellular DAG and TAG content. These data are consistent with findings in adipose tissue for which it has also been shown that the FA composition of the TAG fraction reflects the dietary patterns over a considerable time. ${ }^{20,21}$ It therefore seems that the dietary FA composition affects skeletal muscle FA composition of both DAG and TAG over a relatively short time. At this moment, too little information is available on the impact of changes in muscle FA composition on muscle function, although suggestions have been made that diet-induced modifications in membrane lipid composition are associated with changes in energy metabolism and may impact substantially on diseases such as the metabolic syndrome by influencing a range of glucose- and lipid-handling pathways. ${ }^{35}$ Clearly, more information is needed on the impact of muscle FA composition on muscle metabolism, but our data are encouraging in that respect. On another note, analysis of muscle FA composition could help to test the adherence to diets in short-term nutritional intervention studies, although this would only be applicable in studies including invasive measurements.

An explanation for the differences in muscle fat accumulation between diets could be changes in fat oxidative and mitochondrial capacity. Indeed, in recent years, several studies have shown that a high intake of dietary fat affects mitochondrial oxidative capacity in skeletal muscle. ${ }^{36-41}$ Although the functional analysis of mitochondrial function was out of scope of the present study, we did measure markers of mitochondrial metabolism. Measurement of citrate synthase activity revealed no such differences. $\beta$-Hydroxyacyl-CoA dehydrogenase activity, reflecting 
$\beta$-oxidation capacity, was also similar between the diets, with varying FA composition.

Mitochondrial function was also assessed by measurement of the OXPHOS complexes and PGC1 $\alpha$. However, we did not observe a negative effect of HF feeding on these markers of mitochondrial metabolism. Although we did not directly measure the functional consequences of the different $\mathrm{HF}$ diets, our results are in accordance with some recent studies that also failed to show a negative effect of HF feeding on mitochondrial function. Thus, we showed that an 8-week HF dietary intervention in male Wistar rats did not affect the intrinsic functioning of rat skeletal muscle mitochondria as assessed by respirometry. ${ }^{42}$ Also, Bonnard et al. ${ }^{43}$ did not detect mitochondrial dysfunction after a 4-week HF diet intervention in $\mathrm{C} 57 \mathrm{BL} / 6 \mathrm{~J}$ mice, whereas in an extended dietary intervention of 16 weeks, altered mitochondrial biogenesis, structure and function in muscle tissue were observed. According to Bonnard et al.," ${ }^{43}$ mitochondrial alterations do not precede the onset of insulin resistance. Possibly, this also holds true for our data, but our data do not allow elaboration on insulin resistance.

As mitochondrial oxidative capacity could not explain differences in muscle fat accumulation, we also examined whether the different diets exert beneficial effects on muscle fat balance by stimulating lipolysis. However, the measurement of ATGL, the main lipase responsible for the breakdown of TAG into DAG, was unaffected by diet composition.

In summary, we show that after 8 weeks of HF feeding, the FA profile of both TAG and DAG lipid intermediates in the muscle mimicked the FA profile of the individual HF diets, indicating that after a relatively short period the dietary fat intake relates to the FA composition of the lipid metabolites in the muscle.

Furthermore, we show that a HF diet, rich in SO, is able to prevent accumulation of fat in skeletal muscle. Finally, we could not find evidence for a negative effect of HF diets on markers of mitochondrial metabolism.

\section{Conflict of interest}

The authors declare no conflict of interest.

\section{Acknowledgements}

This study was funded by Top Institute (TI) Food and Nutrition. TI Food and Nutrition, formerly known as WCFS, is a unique public/private partnership that generates vision on scientific breakthroughs in food and nutrition, resulting in the development of innovative products and technologies that respond to consumer demands for safe, tasty and healthy foods. Partners are major Dutch food companies and research organizations. A VICI (Grant 918.96.618) and a VIDI (Grant 917.66.359) for innovative research from the Netherlands Organization for Scientific Research (NWO) support the work of PS and $\mathrm{MH}$, respectively.

\section{References}

1 Frayn KN. Adipose tissue as a buffer for daily lipid flux. Diabetologia 2002; 45: 1201-1210.

2 Starling RD, Trappe TA, Parcell AC, Kerr CG, Fink WJ, Costill DL. Effects of diet on muscle triglyceride and endurance performance. J Appl Physiol 1997; 82: 1185-1189.

3 Helge JW, Wulff B, Kiens B. Impact of a fat-rich diet on endurance in man: role of the dietary period. Med Sci Sports Exerc 1998; 30: $456-461$.

4 Helge JW, Watt PW, Richter EA, Rennie MJ, Kiens B. Fat utilization during exercise: adaptation to a fat-rich diet increases utilization of plasma fatty acids and very low density lipoproteintriacylglycerol in humans. J Physiol 2001; 537: 1009-1020.

5 Jansson E, Kaijser L. Effect of diet on muscle glycogen and blood glucose utilization during a short-term exercise in man. Acta Physiol Scand 1982; 115: 341-347.

6 Goodpaster BH, Kelley DE. Skeletal muscle triglyceride: marker or mediator of obesity-induced insulin resistance in type 2 diabetes mellitus? Curr Diab Rep 2002; 2: 216-222.

7 An J, Muoio DM, Shiota M, Fujimoto Y, Cline GW, Shulman GI et al. Hepatic expression of malonyl-CoA decarboxylase reverses muscle, liver and whole-animal insulin resistance. Nat Med 2004; 10: $268-274$.

8 Hulver MW, Berggren JR, Carper MJ, Miyazaki M, Ntambi JM, Hoffman EP et al. Elevated stearoyl-CoA desaturase-1 expression in skeletal muscle contributes to abnormal fatty acid partitioning in obese humans. Cell Metab 2005; 2: 251-261.

9 Hulver MW, Berggren JR, Cortright RN, Dudek RW, Thompson $\mathrm{RP}$, Pories WJ et al. Skeletal muscle lipid metabolism with obesity. Am J Physiol Endocrinol Metab 2003; 284: E741-E747.

10 Bell RR, Spencer MJ, Sherriff JL. Voluntary exercise and monounsaturated canola oil reduce fat gain in mice fed diets high in fat. J Nutr 1997; 127: 2006-2010.

11 Larson DE, Hunter GR, Williams MJ, Kekes-Szabo T, Nyikos I, Goran MI. Dietary fat in relation to body fat and intraabdominal adipose tissue: a cross-sectional analysis. Am J Clin Nutr 1996; 64: 677-684.

12 Ikemoto S, Takahashi $\mathrm{M}$, Tsunoda $\mathrm{N}$, Maruyama $\mathrm{K}$, Itakura $\mathrm{H}$, Ezaki O. High-fat diet-induced hyperglycemia and obesity in mice: differential effects of dietary oils. Metabolism 1996; 45: 1539-1546.

13 Fernandez S, Gonzalez C, Diaz F, Fueyo AM, Gutierrez JM, Patterson AM. Long-term effects in two generations of enriched soybean and olive oil diets on some cardiovascular and biochemical parameters in male rats. Int J Vitam Nutr Res 1996; 66: 393-399.

14 Doucet E, Almeras N, White MD, Despres JP, Bouchard C, Tremblay A. Dietary fat composition and human adiposity. Eur J Clin Nutr 1998; 52: 2-6.

15 Leyton J, Drury PJ, Crawford MA. Differential oxidation of saturated and unsaturated fatty acids in vivo in the rat. Br J Nutr 1987; 57: 383-393.

16 DeLany JP, Windhauser MM, Champagne CM, Bray GA. Differential oxidation of individual dietary fatty acids in humans. Am J Clin Nutr 2000; 72: 905-911.

17 Gaster M, Rustan AC, Beck-Nielsen H. Differential utilization of saturated palmitate and unsaturated oleate: evidence from cultured myotubes. Diabetes 2005; 54: 648-656.

18 Montell E, Turini M, Marotta M, Roberts M, Noe V, Ciudad CJ et al. DAG accumulation from saturated fatty acids desensitizes insulin stimulation of glucose uptake in muscle cells. Am J Physiol Endocrinol Metab 2001; 280: E229-E237.

19 Schmitz-Peiffer C, Craig DL, Biden TJ. Ceramide generation is sufficient to account for the inhibition of the insulin-stimulated PKB pathway in C2C12 skeletal muscle cells pretreated with palmitate. J Biol Chem 1999; 274: 24202-24210.

20 Hirsch J, Farquhar JW, Ahrens Jr EH, Peterson ML, Stoffel W. Studies of adipose tissue in man. A microtechnic for sampling and analysis. Am J Clin Nutr 1960; 8: 499-511. 
21 Dayton S, Hashimoto S, Dixon W, Pearce ML. Composition of lipids in human serum and adipose tissue during prolonged feeding of a diet high in unsaturated fat. J Lipid Res 1966; 7: 103-111.

22 Schurch AF, Lloyd LE, Crampton EW. The use of chromic oxide as an index for determining the digestibility of a diet. J Nutr 1950; 41: 629-636.

23 Folch J, Lees M, Sloane Stanley GH. A simple method for the isolation and purification of total lipides from animal tissues. J Biol Chem 1957; 226: 497-509.

24 Morrison WR, Smith LM. Preparation of fatty acid methyl esters and dimethylacetals from lipids with boron fluoride - methanol. J Lipid Res 1964; 5: 600-608.

25 Bergmeyer HU, Gawehn K, Grassl M. 3-Hydroxyacyl-CoA dehydrogenase. In: Bergmeyer HU (ed). Methods of Enzymatic Analysis. Verslag Chemie GmbH: Weinheim, Germany, 1974, p 474.

26 Shepard D, Garland PB. Citrate synthase from rat liver. Methods Enzymol 1969; 13: 11-16.

27 Schrauwen P, Hoeks J, Schaart G, Kornips E, Binas B, Van De Vusse GJ et al. Uncoupling protein 3 as a mitochondrial fatty acid anion exporter. FASEB J 2003; 17: 2272-2274.

28 Chen IS, Subramaniam S, Vahouny GV, Cassidy MM, Ikeda I, Kritchevsky D. A comparison of the digestion and absorption of cocoa butter and palm kernel oil and their effects on cholesterol absorption in rats. J Nutr 1989; 119: 1569-1573.

29 Apgar JL, Shively CA, Tarka Jr SM. Digestibility of cocoa butter and corn oil and their influence on fatty acid distribution in rats. J Nutr 1987; 117: 660-665.

30 Couet C, Delarue J, Ritz P, Antoine JM, Lamisse F. Effect of dietary fish oil on body fat mass and basal fat oxidation in healthy adults. Int J Obes Relat Metab Disord 1997; 21: 637-643.

31 Riccardi G, Giacco R, Rivellese AA. Dietary fat, insulin sensitivity and the metabolic syndrome. Clin Nutr 2004; 23: 447-456.

32 Riserus U, Willett WC, Hu FB. Dietary fats and prevention of type 2 diabetes. Prog Lipid Res 2009; 48: 44-51.

33 Summers LK, Fielding BA, Bradshaw HA, Ilic V, Beysen C, Clark ML et al. Substituting dietary saturated fat with polyunsaturated fat changes abdominal fat distribution and improves insulin sensitivity. Diabetologia 2002; 45: 369-377.

34 Morino K, Neschen S, Bilz S, Sono S, Tsirigotis D, Reznick RM et al. Muscle-specific IRS-1 Ser $\rightarrow$ Ala transgenic mice are protected from fat-induced insulin resistance in skeletal muscle. Diabetes 2008; 57: 2644-2651.
35 Hulbert AJ, Turner N, Storlien LH, Else PL. Dietary fats and membrane function: implications for metabolism and disease. Biol Rev Camb Philos Soc 2005; 80: 155-169.

36 Simoneau JA, Veerkamp JH, Turcotte LP, Kelley DE. Markers of capacity to utilize fatty acids in human skeletal muscle: relation to insulin resistance and obesity and effects of weight loss. FASEB J 1999; 13: 2051-2060.

37 Chanseaume E, Malpuech-Brugere C, Patrac V, Bielicki G, Rousset $\mathrm{P}$, Couturier $\mathrm{K}$ et al. Diets high in sugar, fat, and energy induce muscle type-specific adaptations in mitochondrial functions in rats. J Nutr 2006; 136: 2194-2200.

38 Sparks LM, Xie H, Koza RA, Mynatt R, Hulver MW, Bray GA et al. A high-fat diet coordinately downregulates genes required for mitochondrial oxidative phosphorylation in skeletal muscle. Diabetes 2005; 54: 1926-1933.

39 Tunstall RJ, Cameron-Smith D. Effect of elevated lipid concentrations on human skeletal muscle gene expression. Metabolism 2005; 54: 952-959.

40 Iossa S, Lionetti L, Mollica MP, Crescenzo R, Botta M, Barletta A et al. Effect of high-fat feeding on metabolic efficiency and mitochondrial oxidative capacity in adult rats. Br J Nutr 2003; 90 953-960.

41 Obici S, Wang J, Chowdury R, Feng Z, Siddhanta U, Morgan K et al. Identification of a biochemical link between energy intake and energy expenditure. J Clin Invest 2002; 109: 1599-1605.

42 Hoeks J, Briede JJ, de Vogel J, Schaart G, Nabben M, MoonenKornips $\mathrm{E}$ et al. Mitochondrial function, content and ROS production in rat skeletal muscle: effect of high-fat feeding. FEBS Lett 2008; 582: 510-516.

43 Bonnard C, Durand A, Peyrol S, Chanseaume E, Chauvin MA, Morio B et al. Mitochondrial dysfunction results from oxidative stress in the skeletal muscle of diet-induced insulin-resistant mice. J Clin Invest 2008; 118: 789-800.

This work is licensed under the Creative Commons Attribution-NonCommercial-No Derivative Works 3.0 Unported License. To view a copy of this license, visit http://creativecommons.org/ licenses/by-nc-nd/3.0/ 